\title{
THE ROLE OF DRIFT ON DIURNAL ANISOTROPY OF GALACTIC COSMIC RAYS IN DIFFERENT PERIODS OF SOLAR MAGNETIC CYCLES
}

\section{Iskra, Krzysztof}

Siedlce University of Natural Sciences and Humanities, Address, Str. Konarskiego 2, 08 -110 Siedlce, Poland

E-mail: iskra@uph.edu.pl

\section{Alania, Michael}

Siedlce University of Natural Sciences and Humanities Address, Str. Konarskiego 2, 08 -110 Siedlce, Poland

Ivan Javakhishvili Tbilisi State University, Nodia Institute of Geophysics,

E-mail: alania@uph.edu.pl

\section{Modzelewska, Renata}

Siedlce University of Natural Sciences and Humanities

Address, Str. Konarskiego 2, 08 -110 Siedlce, Poland

E-mail: renatameuph.edu.pl

\section{Siłuszyk, Marek}

Siedlce University of Natural Sciences and Humanities Address, Str. Konarskiego 2, 08 -110 Siedlce, Poland

E-mail: marek.siluszyk@uph.edu.pI

\section{Wozniak, Witold}

Polish Oil and Gas Company, Warsaw, Poland Address, 25 M. Kasprzaka Str, 01-224 Warsaw, Poland E-mail: witek.wozniakegmail.com

\section{Wolinski, Pawel}

Warsaw University of Technology, Warsaw, Poland Address, Plac Politechniki 1, 00-661 Warsaw, Poland E-mail: pawxxepoczta.onet.pl 
The hourly neutron monitor data have been used to study the role of drift effect in the temporal changes of the diurnal anisotropy. In order to thoroughly separate sectors of the Interplanetary Magnetic Field (IMF) and its influence on the anisotropy of Galactic Cosmic Rays (GCRs) for positive $(A>0)$ and negative $(A<0)$ polarities of solar magnetic cycle, two periods $(1995-1997$ $(A>0)$ and 2007-2009 $(A<0)$ ) have been considered. We study drift effects in diurnal anisotropy of GCR caused by the gradient and curvature of the regular IMF, and due to the heliospheric neutral sheet. We use the harmonic analyses method to calculate radial Ar and tangential A $\varphi$ components of the ecliptic diurnal anisotropy of GCR based on data of NM for cut-off rigidities less than $5 \mathrm{GV}$. It is shown that there are differences between the diurnal anisotropy of GCR found in the reliably established various sectors (duration of each sectors is $\geq 4$ days) of IMF. An interpretation of obtained results are provided based on the present modern theory of GCR propagation.

The 34th International Cosmic Ray Conference

30 July- 6 August, 2015

The Hague, The Netherlands 


\section{Introduction}

Investigation of the influence of the sector structure of the interplanetary magnetic field (IMF) and the global magnetic field of the Sun on the diurnal anisotropy of galactic cosmic rays (GCR) has remained one of the interesting aspects of GCR modulation. First of all, the reliable manifestation of drift effect in the diurnal anisotropy of GCR is important for the theory of GCR modulation, according to which drift play a significant role in the understanding of the features of GCR behavior in the heliosphere [1-5]. On the other hand, if drift in the diurnal anisotropy of GCR is established successfully, it can be used for calculations of the various parameters characterized the solar wind and the diffusion of GCR. The system of equations for the components of the diurnal anisotropy of GCR [6-9] has the following form:

$$
\begin{aligned}
& A_{r}^{ \pm}=3\left[C U-\mathrm{K}_{r r} G_{r}^{ \pm} \pm \mathrm{K}_{T} G_{\theta}^{ \pm} \sin \Psi+\left(\mathrm{K}_{\amalg}-\mathrm{K}_{\perp}\right) G_{\varphi}^{ \pm} \sin \Psi \cos \Psi\right] / V \\
& A_{\theta}^{ \pm}=-3\left[ \pm \mathrm{K}_{T} G_{r}^{ \pm} \sin \Psi+\mathrm{K}_{\perp} G_{\theta}^{ \pm} \pm \mathrm{K}_{T} G_{\varphi}^{ \pm} \cos \Psi\right] / V \\
& A_{\varphi}^{ \pm}=3\left[\left(\mathrm{~K}_{\amalg}-\mathrm{K}_{\perp}\right) G_{r}^{ \pm} \sin \Psi \cos \Psi \pm \mathrm{K}_{T} G_{\theta}^{ \pm} \cos \Psi-\mathrm{K}_{\varphi \varphi} G_{\varphi}^{ \pm}\right] / V
\end{aligned}
$$

Where, (+) and (-) correspond to the IMF lines away from (positive) and toward (negative) the northern hemisphere of the Sun. Here, $\mathrm{K}_{\mathrm{U}}, \mathrm{K}_{\perp}$ and $\mathrm{K}_{T}$ are parallel, perpendicular and drift diffusion coefficients, respectively, of GCR with respect to the interplanetary magnetic field lines. $G_{r}^{ \pm}, G_{\theta}^{ \pm}$and $G_{\varphi}^{ \pm}$are the radial, heliolatitudinal and heliolongitudinal gradients of GCR in the interplanetary space; $\psi$ is the angle between the IMF lines and the Earth-Sun line; U and V are speeds of the solar wind and of the GCR particles, respectively. The Compton-Getting factor $\mathrm{C}$ is equal to $\sim 1.5$ for GCR sensitive to neutron monitors.

On the average, the diurnal anisotropy of GCR is about $0.3-0.4 \%$ according to neutron monitors data and a contribution of the effect of drift in it may reach $0.05-0.1 \%[7,10]$ which coincides with the modelling results of [10] and [11]. It should be noticed about two kinds of drift effects in the diurnal anisotropy of GCR. The first one, stipulated by the gradient and curvature of the global magnetic field of the Sun, can be clearly manifested in the average diurnal anisotropy of GCR for the different solar magnetic cycles A>0 (1971-1979 and 19911999) and $A<0$ (1959-1970 and 1981-1989). In these case owing to the averaging for a long period (much greater than the duration of the sectors of the IMF) a drift effect in the diurnal anisotropy due to the sector structure of the IMF becomes negligible. A source of the second kind of drift effect in the diurnal anisotropy of GCR is an existence of the heliospheric neutral sheet (HNS) of the IMF. So that, the second type of drift considered here, generally is the HNS drift in $[1,3,4]$. We assume that it can be manifested due to the sector structure of IMF for the minima epochs of solar activity, when the tilt angles of the HNS are $<10-15$ degrees, i.e. the Earth is located near the weakly wavy HNS. So, for the reliable manifestation of the first type of the drift in the diurnal anisotropy of GCR it is sufficient to average data for long time of observation. At the same time, a single-valued manifestation of the influence of the sectors of the IMF on the diurnal anisotropy is a complicated problem on the background of the significant temporal changes of the solar wind parameters lasting comparable with the duration of the positive and negative sectors of IMF. So, in order to manifest the drift effect caused by the sector structure of IMF in the diurnal anisotropy of GCR one has to consider the minima epochs of solar activity. In the minima epochs the temporal changes of the solar wind parameters and the variations of GCR associated with the Sun's rotation are relatively negligible, while the sector structure of IMF is clearly manifested. 
In the paper [7] the equations (1) to (3) were used to calculate the various parameters, which characterized the solar wind, and the diffusion coefficients of GCR supposing the equality of the radial and transversal gradients $G_{r}^{ \pm}$and $G_{\theta}^{ \pm}$in different sectors of IMF for the minima epochs of solar activity. The components of diurnal anisotropy of GCR $A_{r}^{ \pm}$and $A_{\varphi}^{ \pm}$were calculated using harmonic analyses. The same problem was considered in [12]. In papers [18-20] the components of diurnal anisotropy of GCR $A_{r}^{ \pm}$and $A_{\varphi}^{ \pm}$were calculated using the global survey method.

\section{Experimental results and discussion. Drift effect in the diurnal anisotropy of GCR}

A purpose of this paper is to carry on a study of the role drift effect in the temporal changes of the diurnal anisotropy in two sequential minima and near minima periods (1995-1997 (A>0) and 2007-2009 $(\mathrm{A}<0)$ ) The hourly neutron monitor data with magnetic cut-off rigidities $\leq 5 \mathrm{GV}$ have been used. In doing so, the days with amplitudes more than $0.7 \%$ were excluded from all neutron monitors data, considering them as the anomalous diurnal variation caused by strong disturbances in interplanetary space. The data of the sector structure of the IMF was taken from direct observations of satellites in [13]. Besides the calculations of the $A_{r}$ and $A_{\varphi}$ components of the diurnal anisotropy of GCR have been done for the positive and negative sectors of IMF with a duration $\geq 4$ days. Base on the ordinary harmonic analysis the components $A_{r}$ and $A_{\varphi}$ of the

diurnal anisotropy of GCR were calculated. In figure 1 are plotted the vector diagrams of the diurnal anisotropy of GCR in percent for different sectors ('+' corresponds to the positive and '-' to the negative directions of the IMF) for the periods 1995, 1996, 1997 -positive (A>0) polarity and 2007, 2008, 2009 -negative $(A<0)$ polarity of solar magnetic cycle.

One can see that there are differences between the amplitudes and phases for different sectors of the IMF in the above-mentioned two solar minima and near minima epochs. The influence on the diurnal anisotropy of GCR of the sector structure of the IMF in the minima and near minima epochs of solar activity (when the tilt angles of the HNS are not too large) is generally caused by the HNS drift and has mostly an azimuthal direction (see Figure 1). In the period 1995, 1996, 1997 (minimum and near minimum for $A>0$ ) the anisotropy vector for the positive sectors in the relation to the anisotropy vector for negative sectors is slightly shifted towards the earlier hours. In the period 2007, 2008, 2009 (minimum and near minimum for $\mathrm{A}<0$ ) the anisotropy vector for the positive sectors have an opposite tendency. It is shifted in the relation to the anisotropy vector for negative sectors towards the later hours.

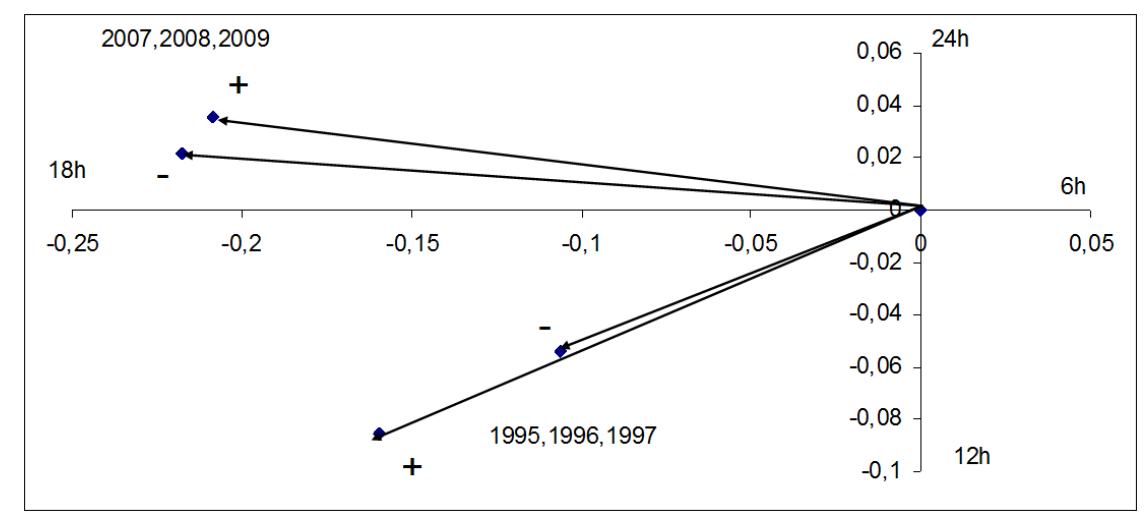

Figure 1. Harmonic diagram of the anisotropy for positive (+) and negative (-) sectors of IMF. 
On the vertical and horizontal axes are the radial and azimuthal components, in $\%$.

It seems that on the drift effect of the anisotropy in different sectors of the IMF influence the features of the heliolatitudinal gradient of GCR caused by the heliolatitudinal asymmetry of the heliosphere [14].

In figure 2 are plotted averaged anisotropies of GCR for the same polarity for 1995, 1996, 1997 -positive and 2007, 2008, 2009-negative direction of the global magnetic field. The vector of anisotropy for the period $\mathrm{A}>0$ tends to earlier hours and the vector of anisotropy for period $\mathrm{A}<0$ tends to later hours in relation to 18 hour.

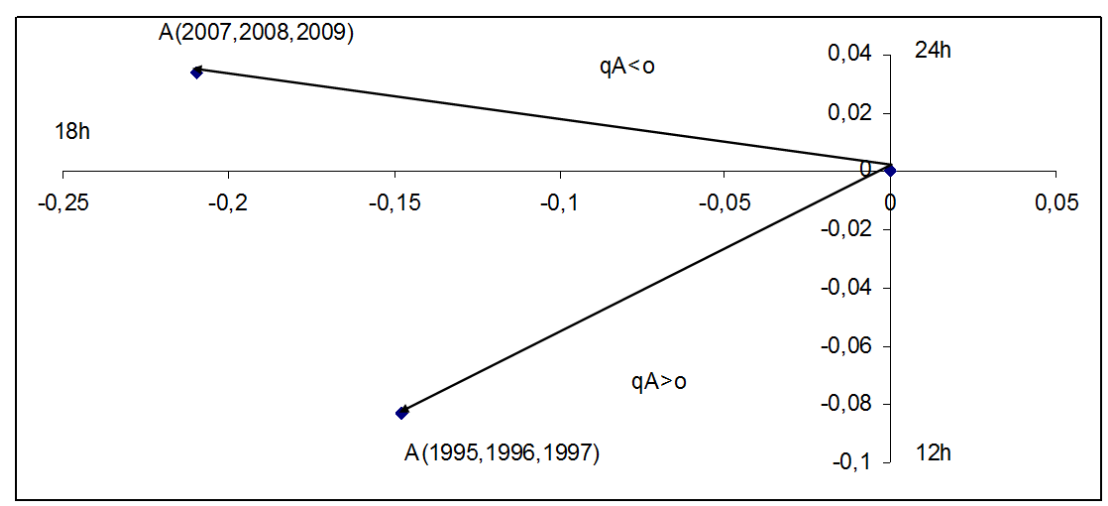

Figure 2 Harmonic diagram of the averaged anisotropy for 1995, 1996, 1997 -positive and 2007, 2008, 2009 -negative direction of global magnetic field.

Let $\mathrm{A}$ is the averaged amplitude of the diurnal anisotropy for both of the $\mathrm{A}>0$ and the $\mathrm{A}<0, \mathrm{~A}=$ $[\mathrm{A}(1995,1996,1997)+\mathrm{A}(2007,2008,2009)] / 2$, from which the drift effect is excluded. Average vectors $A(1995,1996,1997)$ and $A(2007,2008,2009)$ are presented in Figure 3. The drift vectors $\mathrm{A}(+)=(\mathrm{A}(1995,1996,1997)-\mathrm{A})$ and $\mathrm{A}(-)=(\mathrm{A}(\mathrm{A}) 2007,2008,2009)-\mathrm{A})$, are also shown in figure 3 . One can see that for the $A>0$ magnetic cycle the radial component of the anisotropy vector of $\mathrm{A}(+)$ is directed to the Sun (to the direction of 12 hours in the harmonic diagram), and for the $\mathrm{A}<0$ cycle the radial component of the anisotropy vector of $\mathrm{A}(-)$ is directed out of the Sun (to the direction of $24 \mathrm{~h}$ in the harmonic diagram). These experimental results are in agreement with the drift theory [15] of modulation of GCR according to which in the A>0 cycle drift stream of GCR caused by the gradient and curvature of the IMF is preferentially coming from the polar regions to the helio-equatorial vicinity and is directed away from the Sun. The opposite direction of the drift stream of GCR exists for the A $<0$ cycle. Thus, the drift anisotropy vectors $\mathrm{A}(+)$ and $\mathrm{A}(-)$ of GCR (caused by the gradient and curvature of the IMF) basically contain the radial component (see Figure 3), while the effect of drift due to the sector structure of the IMF is generally caused by the HNS and is mostly manifested in the azimuthal component of the diurnal anisotropy of GCR (see Figure 1).

The difference between amplitudes $\mathrm{A}(\mathrm{A}>0)$ and $\mathrm{A}(\mathrm{A}<0)$ caused by the drift of GCR due to the gradient and curvature of the regular IMF and is the general source of the 22-year variation of the diurnal anisotropy of GCR measured by neutron monitors and meson telescopes in $[16,17]$. 


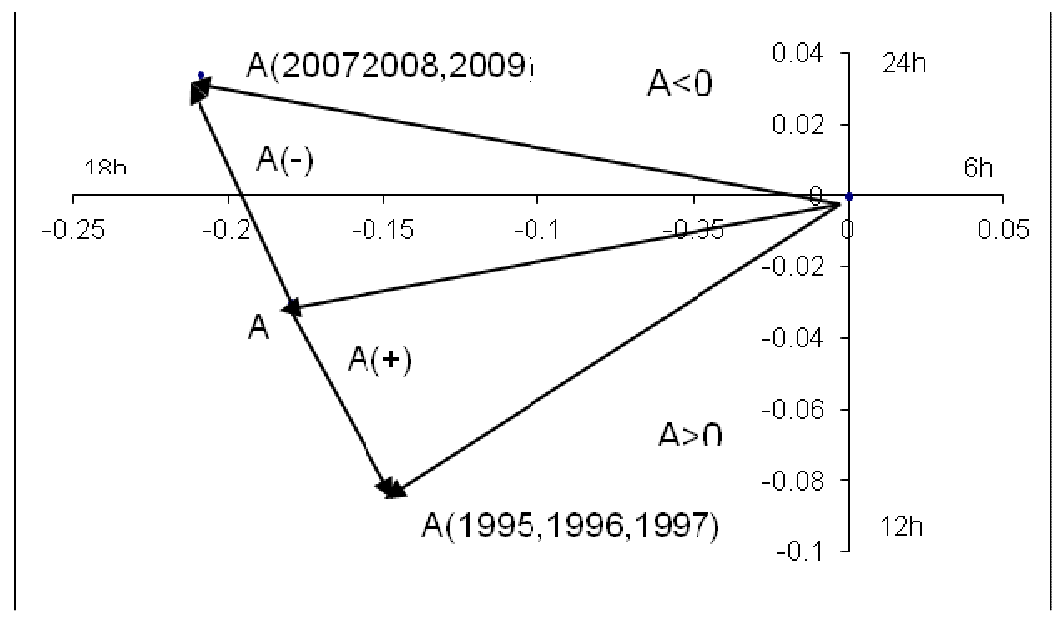

Figure 3 Harmonic diagram of the anisotropy $\mathrm{A}(1995,1996,1997)$ for the $\mathrm{A}>0$ and $\mathrm{A}(2007$, $2008,2009)$ for the $A<0$ solar magnetic cycles, averaged amplitude of the diurnal anisotropy $A$ for both of the $\mathrm{A}>0$ and the $\mathrm{A}<0$ and drift vectors $\mathrm{A}(+)=(\mathrm{A}(1995,1996,1997)-\mathrm{A})$ and $\mathrm{A}(-)=(\mathrm{A}(2007,2008,2009)-\mathrm{A})$.

\section{Acknowledgment}

Authors thank the investigators of websites:

http://spidr.ngdc.noaa.gov/spidr,http://134.245.132.179

http://www.ngdc.noaa.gov/stp/SOLAR/ftpsunspotnumber

http://wso.stanford.edu/SB/SB.Svalgaard.html

http://www.izmiran.ru/

\section{Conclusions}

1. Drift effects in the diurnal anisotropy of GCR caused by the gradient and curvature of the global solar magnetic field is manifested in the radial component of the diurnal anisotropy. For the $\mathrm{A}>0$ magnetic cycle the radial component of the diurnal anisotropy is directed to the Sun, while for the $\mathrm{A}<0$ cycle the radial component of diurnal anisotropy is directed away from the Sun. This type of drift effect is the source of the 22-year variation of the diurnal anisotropy of GCR.

2. The effect of drift in the diurnal anisotropy of GCR due to the sector structure of the IMF during the minima epochs of solar activity is generally caused by the HNS and is mostly pronounced in the azimuthal component.

\section{References}

[1] Kota, J., and J. R. Jokipii, Effects of drift on the transport of cosmic rays. VI. A three-dimensional model including diffusion, Astrophys. J., 265,573, 1983.

[2] Kota, J., and J. R. Jokipii, Modelling of 3-D cosmic ray corotating structure in the heliosphere, Space Sci. Rev., 83, 137, 1998.

[3] Potgieter, M. S., and H. Moraal, A drift model for the modulation of galactic cosmic rays, 
Astrophys. J., 294, 425,1985.

[4] Burger, R. A., and M. S. Potgieter, The calculation of neutral sheet drift in two-dimensional cosmic-ray modulation models, Astrophys. .I, 339, 501, 1989.

[5] Potgieter, M. S. The long-term modulation of galactic cosmic rays in the heliosphere, Adv. Space Res., 16(9), 191,1995.

[6] Krymski, G. F., Diffusion mechanism of diurnal cosmic-ray variation, Geomagn. Aeronomy, 4, $763,1964$.

[7] Alania, M. V., R. G. Aslamazashvili, T.V. Djapiashvili et al., 18 ICRC, Con. Papers. 10, 91, 1983.

[8] Riker, J. F., and H. S. Ahluwalia, A survey of cosmic ray diurnal variation during 1973-79, 2, Application of diffusion-convection model to diurnal anisotropy data, Planet. Space Sci., 35,1117, 1987.

[9] Bieber, J. W., and J. Chen, Cosmic ray diurnal anisotropy, 1936-1988: Implications for drift and modulation theories, Astrophysical J., 372, 30 1, 1991.

[10] Alania, M. V., and T. V. Djapiashvili, Proc. 16thInter Cosmic Ray Conf, 3,19, 1979.

[11] Potgieter, M. S,. and M. L. Van Staden, The effect of the changing polarity and neutral sheet of the IMF on the cosmic ray diurnal anisotropy at neutron monitor energies, Planet. Space Sci., 39(9), 1233, 1991.

[12] Ahluwalia, N. S., and L. I. Dorman, Proc. Inter Cosmic Ray Conf, 2,4-6, 101,1997.

[13] http://wso.stanford.edu/SB/SB.Svalgaard.html

[14] Smith, E. J., J. R. Jokipii, J. Kota, R. P. Lepping, and A. Szabo, Evidence of a north-south asymmetry in the heliosphere associated with a southward displacement of the heliospheric current sheet, Astrophys. J., 353,1084,2000.

[15] Jokipii, J. R., E. H. Levy, and W. B. Hubbard, Effects of particle drift on cosmic-ray transport. 1. General properties, Application to Solar Modulation, Astrophys. J., 213, 861, 1977.

[16] Forbush, S. E., Variation with a period of two solar cycles in cosmic ray diurnal anisotropy and the superposed variations correlated with magnetic activity, J. Geophys. Res.,74,345 1, 1969.

[17] Ahluwalia, N. S., Is there a twenty year wave in the diurnal anisotropy of cosmic rays?, Geophys. Rex Lett., 3,287, 1988.

[18] Alania M.V., Aslamazashvili R.G.,Bochirishvili T.B.,Iskra K., Siluszyk M., The role of drift on diurnal anisotropy and on temporal changes in the energy spectra of the 11-year variation for galactic cosmic rays,//Adv. Space Res. -2001. - 27, 3. -P. 613-618.

[19] Alania M.V.,Bochorishvili T.B., Iskra K. Effects of the Sector Structure of the Interplanetary Magnetic Field on Galactic Cosmic Ray Anisotropy // Solar System Research. -2003. -v.37, Issue 6. -P. 519-522

[20] Iskra K, Modzelewska R.,Siluszyk M., and Alania M.V. Features of long -term variations of galactic cosmic ray intensity and anisotropy // Kinematics and Physics of Celestial Bodies. 2006. - Т.22, № 6. -P.402-412. 